\title{
Diversities in the Interpretation of the p-n Junction Under All Injection Conditions
}

\author{
Mohammad Asadul Haque* and Jalalur Rahman \\ Department of Applied Physics, Electronics and Communication Engineering, \\ University of Dhaka,Dhaka-1000, Bangladesh
}

\begin{abstract}
A comprehensive theory of the physical picture of a p-n junction under all injection conditions has been presented in this paper. The pn product in a junction must always be $\leq n_{i}^{2} e^{\frac{q V}{k T}}$. By incorporating all suitable modification of the boundary conditions, the inconsistencies concerning the relation of the applied potential difference to the difference across the space charge region of a p-n junction were observed. Attempt were made to remove those inconsistencies. The transition region for abrupt p-n junction is analyzed in terms of carrier densities at the neutral bulk-space charge region boundaries and location of these boundaries with respect to the metallurgical junction and also analyzed by using quasi-equilibrium assumptions. The p-n junction is solved by using three-region approach and analytical solutions are derived for low, medium and high injections.
\end{abstract}

Key words: Depletion region, Injection conditions, Quasi-Fermi-level Inconsistencies.

\section{Introduction}

When a piece of p-type material is in physical contact with n-type material then the interface between the different regions is called p-n junction. The basic theory of p-n junction was established by Shockley (Kasap S. O., 1990-2001) on the basis of four assumptions. The S-R-H theory (Shockley W. et al. 1952, Hall R. N., 1952) is established on the basis of generation and recombination phenomenon. Rittner (Rittner E. S. 1964, Hazman S., 1967 ) studied on Shockley model and suggested that model of Shockley is applicable to the emitter and collector regions for all realizable values of current, where as it is applicable to the base region only for extremely small currents. Misawa (Misawa T., 1967) put forward the boundary condition to understand the p-n junction model that the Boltzmann factor and the electrochemical potential approach at the injection surface and the pn product of $\mathrm{p}-\mathrm{n}$ junction must always be $\leq n_{i}^{2} e^{\frac{q V}{k T}}$ outside the depletion region. However Fletcher (Fletcher N. H., 1957) showed that in high injection conditions, the quasi-Fermi levels separated by an amount larger than the voltage drop $\mathrm{V}_{\mathrm{J}}$ appearing across the junction. By using the above boundary conditions attempt has been taken to understand the $\mathrm{p}$-n junction from different approach. van Vliet (van Vliet K. M., 1984) suggested that the basic high

\footnotetext{
* Corresponding author: E-mail: asad2310@yahoo.com
}

injection effects should include some conditions and modified S-R-H theory on the basis of that condition.

Nordman (Nordman J. E.,1967) agreed with results of van Vliet with only one exception by using three guide lines. Nussbaum (Nussubaum A., 1978) reported about the inconsistencies and tried to remove these inconsistencies by using correction factor. Guckel et al. (Guckel H. et al. 1989) analyzed p-n junction by using quasi-equilibrium assumption and the results cover three injection conditions. The p-n junction its depletion layer, its behavior under several injection conditions etc have been analyzed in different approach by several scientists since its very inception; the results are agreements and disagreements. The purpose of this paper is to attempt to focus on all points and achieve to find a modified picture for understanding of a p-n junction.

\section{Diversities in the interpretation of the p-n junction}

The basic theory of p-n junction do not give clear idea about depletion region of a p-n junction as a function of carrier concentration, the carrier lifetime and diffusion constant in equilibrium situation. 
Moreover, the concept and assumption of basic theory also do not give any idea about the behavior of a $p$-n junction under all injection conditions in non-equilibrium situation. The S-R-H theory is established on the basis of generation and recombination phenomena

$$
(G-R)_{S R H}=\frac{n p-n_{i}^{2}}{\left(n+n_{1}\right) \tau_{p o}+\left(p+p_{1}\right) \tau_{\text {no }}}
$$

van Vliet (van Vliet K. M., 1984) suggested that the basic high injection effects should include the incorporation of an electric field or in charge neutrality approximation of self consistent field should be considered, there should be incorporation of charge replenishment (injection or extraction) ohmic contacts and the voltage drop the $\mathrm{n}$ and $\mathrm{p}$ regions should exists. It could be modified the S-R-H theory at high injection condition shown that

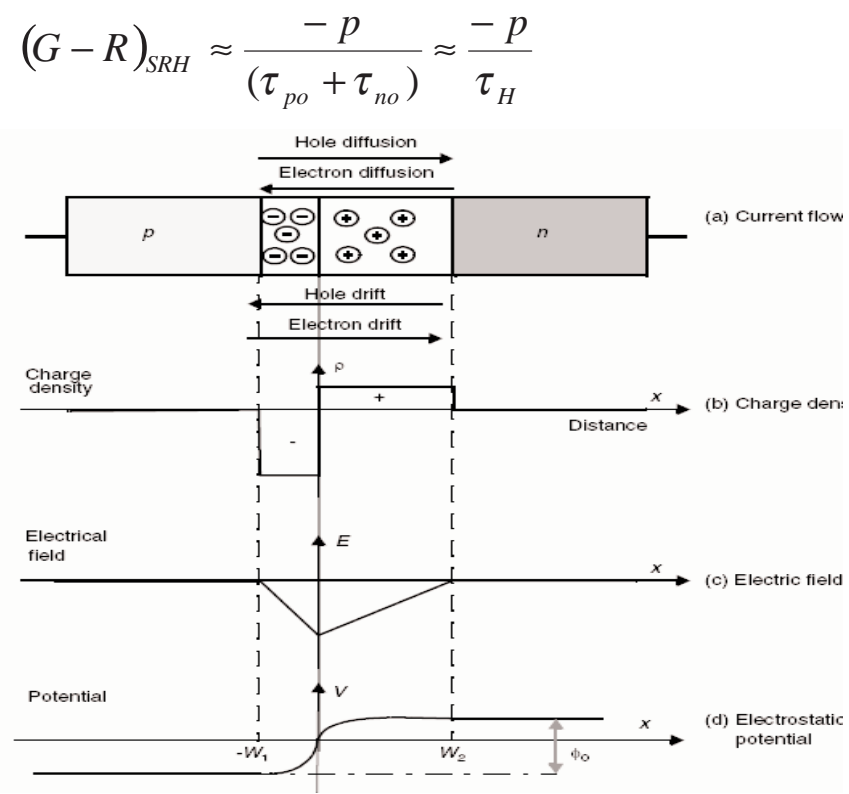

Fig: 1 The abrupt pn-junction under equilibrium bias

Nordman (Nordman J. E., 1967) agreed well with the results of van Vliet and he mentioned three guide lines that are at the ohmic contact the quasi-Fermi levels and equilibrium Fermi level will coincide; the quasi-Fermi levels are constant throughout the transition region; the individual particle current densities can always be given by

$$
J_{p}=q \mu_{p} \operatorname{pgradE}_{F P} \text { and }
$$

$J_{n}=q \mu_{n} \operatorname{ngrad}_{F n}$ for the non degenerate situation. Nussbaum (Nussubaum A., 1978) reported that the contro- versy concerning the relation of the applied potential difference to the difference across the space charge region of a $\mathrm{p}-\mathrm{n}$ junction is due to neglecting of non-zero gradients of the electrochemical potentials. By incorporating all these above and by a suitable modification of the Flatcher boundary conditions (Fletcher N. H., 1957) he deduced the following inconsistencies.

$\bar{\mu}_{n N}-\bar{\mu}_{p P}=-q V_{J}$

and $p_{N} n_{N}=\frac{n_{i}^{2} e^{\frac{-q V_{J}}{k T}\left[1+\left\{\frac{n_{o N}}{p_{o P}}+\frac{p_{o P}}{n_{o N}}\right\} x^{2}\right]}}{\left(1-x^{2}\right)^{2}}$

Where

$$
\begin{aligned}
& \mathrm{x}=e^{\left(\frac{-q\left(V_{J}-V_{o}\right)}{k T}\right)} \\
& p_{N}=x p_{p}, n_{p}=x n_{N}
\end{aligned}
$$

Neglecting term $\mathrm{x}^{2}$ and the above equation can be shown to be $\mathrm{p}_{\mathrm{N}} \mathrm{n}_{\mathrm{N}}>n_{i}^{2} e \frac{-q V_{J}}{k T}$. It could be removed the inconsistencies by using the following correction factor-

$\bar{\mu}_{n N}-\bar{\mu}_{p P}=-\epsilon-q V_{J}$

and $p_{N} n_{N}=n_{i}^{2} e^{\left(\frac{\mu_{n P}-\mu_{p N}}{k T}\right)}$

Where

$$
\begin{aligned}
\in= & \bar{\mu}_{n N}-\bar{\mu}_{p P} \\
\epsilon= & k T \log \left(\frac{n_{N}}{n_{P}}\right)+q V_{o}-q V_{J} \\
& n_{P}=x^{\prime} n_{N}, x^{\prime}=e^{\frac{q V_{J}-q V_{o}-\epsilon}{k T}}, p_{N}=x^{\prime} p_{P}
\end{aligned}
$$

Guckel et al. (Guckel H. et al. 1989) analyzed the p-n junction transition region on the basis of quasi-equilibrium assumption: the transition region of a p-n junction cannot be analyzed by assuming constant quasi-Fermi levels without generating paradox; the constant quasi-Fermi level assumption demands that an applied diode bias must appear in to across the transition. The results cover the following injection conditions: the majority carrier contributions at the transition region boundaries are negligible; the changes in transition region width will cause the doping level at the boundaries to change, which in turn causes apparent change in the contact potential; injection condition involves the bulk 
behavior and the statement $V_{J} \sim V_{X}$ must be valid in order to claim that $q V_{x} \approx\left(E_{F N}-E_{F P}\right)$.

De Mari (Mari De., 1997) showed a numerical iterative method of solution of the one-dimensional basic two-carrier transport equations describing the behavior of semiconductor junctions under both steady-state and transient conditions. The method is of a very general character: none of the conventional assumptions and restrictions is introduced and freedom is available in the choice of the doping profile, recombination-generation law, mobility dependencies, injection level, and boundary conditions applied solely at the external contacts.

For a specified arbitrary input signal of either current or voltage as a function of time, the solution yields terminal properties and all the quantities of interest in the interior of the device (such as carrier densities, electric field, electrostatic potential, particle and displacement currents) as functions of both position and time.

Mukul Agrawal (Mukul Agrawal, 2002) without using the quasi neutrality presented the minority carrier recombination lifetime is inversely proportional to the majority carrier equilibrium concentration. More majority carriers mean that minority carriers would relax faster making the lifetime smaller.

\section{Theoretical analysis and assumptions for p-n junc- tion depletion region}

Certain assumptions are made to simplify the problems of the p-n junction:

1. The p-n junction is abrupt, non-degenerate and uniformly doped.

2. The mobilities $\mu_{n}$ and $\mu_{p}$ are constant within each side of the junction and independent of electric field.

3. The junction is in a d. c. steady state condition under forward bias.

4. The contact regions are ohmic and do not permit excess carrier densities.
5. The quasi-Fermi levels in the transition region are constant.

6. Recombination currents in the bulk region with reasonable S-R-H recombination parameters and short physical lengths are small compared to the injected currents. Bulk recombination will therefore be neglected in the determination of the injection efficiency of the junction.

7. The p-n junction is long enough to divide into three regions; two charge neutrally in the bulk is a solution of the majority carrier transport equation and is valid at all injection levels.

8. In forward bias, minority carriers are injected into quasineutral regions and injected minority carriers recombine at the surface (contacts).

9. In reverse bias, minority carriers are extracted from the quasi-neutral regions and extracted minority carriers are generated at the surface (contacts).

The diode behavior has been explained by a three-region analysis: low, medium and high injection.

In low injection, the majority carrier concentrations are not effected by the injection. Almost all the applied voltage is dropped across the transition region. The bulk voltage drops are negligible. Bulk recombination currents do not affect carrier distributions when bulk widths are small compared to the diffusion lengths of the minority carriers. The transition recombination current usually dominates the injected current. As the injection level increases, the significance of the former decreases and thus the injection efficiency increases. The approximate analytic solution for low injection region:

$0 \leq \frac{q V_{x}}{k T} \leq 2 \ln \frac{N_{D}}{n_{i}}$

In that case $\frac{q V_{J}}{k T}=\frac{q V_{x}}{k T}$

In the medium injection region, the lightly doped is conductivity modulated. In this case the majority carrier concentration increases to keep up with the minority carrier injection and maintains charge neutrality. As the majority carrier concentration increases, the bulk injection into the heavily 
doped side increases. Then the injection efficiency deteriorates rapidly. The externally applied voltage is shared by the transition region and the lightly doped bulk region. Although the transition region recombination currents still exist, it is generally negligible compared to the injected currents. Similarly the bulk recombination currents could be negligible.

The approximate analytic solution for medium injection region:

$2 \ln \frac{N_{D}}{n_{i}} \leq \frac{q V_{x}}{k T} \leq 2 \ln \frac{N_{A}}{n_{i}}$

In that case $\frac{q V_{J}}{k T}=\frac{q V_{x}}{k T}+\ln \frac{N_{D}}{n_{i}}$

In the high injection region, the total potential across the transition region approaches to zero, since the external voltage across the transition region, $\mathrm{V}_{\mathrm{J}}$ cannot exceed the built in voltage. The energy bands are now flattened and the injection behaves more like a conductivity-modulated resistor than a diode. The external voltage, less the built in potential, is now dropped across the bulk regions. Both sides of the junction are conductivity modulated and the background doping levels are swamped out by the injection carriers.

The approximate analytic solution for high injection region

$$
\frac{q V_{x}}{k T} \geq 2 \ln \frac{N_{A}}{n_{i}}
$$

In that case $\frac{q V_{J}}{k T} \approx \ln \frac{N_{A} N_{D}}{n_{i}^{2}}$

\section{Results and Discussion}

On the basis of theoretical analyses and by taking constant parameters shown in the Table I different graphs are illustrated. Here mobility parameters for low, medium and high injection region have been calculated by using corresponding equation and Table II.
Table-I

\begin{tabular}{|l|l|l|l|}
\hline $\begin{array}{l}\text { Parameters } \\
\left(\mathrm{cm}^{2} / \mathrm{v}-\mathrm{s}\right)\end{array}$ & $\begin{array}{l}\text { Low } \\
\text { injection } \\
\text { region }\end{array}$ & $\begin{array}{l}\text { Medium } \\
\text { injection } \\
\text { region }\end{array}$ & $\begin{array}{l}\text { High } \\
\text { injection } \\
\text { region }\end{array}$ \\
\hline$\mu_{\mathrm{nn}}$ & 964 & 639 & 292 \\
\hline$\mu_{\mathrm{np}}$ & 292 & 292 & 292 \\
\hline$\mu_{\mathrm{pn}}$ & 400 & 292 & 104 \\
\hline$\mu_{\mathrm{pp}}$ & 104 & 104 & 104 \\
\hline
\end{tabular}

Table-II

\begin{tabular}{|l|l|l|l|}
\hline Parameters & $\begin{array}{l}\text { Constant } \\
\text { Values }\end{array}$ & Parameters & $\begin{array}{l}\text { Constant } \\
\text { Values }\end{array}$ \\
\hline $\mathrm{N}_{\mathrm{A}}$ & $1 \times 10^{18} / \mathrm{cm}^{2}$ & $\mathrm{D}$ & $\mathrm{kT} \mu / \mathrm{q}$ \\
\hline $\mathrm{N}_{\mathrm{D}}$ & $1 \times 10^{16} / \mathrm{cm}^{2}$ & $\mathrm{~W}_{\mathrm{p}}=\mathrm{W}_{\mathrm{n}}$ & $\begin{array}{l}3 \\
\text { micrometer }\end{array}$ \\
\hline $\mathrm{n}_{\mathrm{i}}$ & $\begin{array}{l}1.45 \mathrm{X} 10^{10} \\
/ \mathrm{cm}^{2}\end{array}$ & $\mathrm{a}$ & $\begin{array}{l}1.06 \times 10^{-12} \\
\mathrm{~F} / \mathrm{cm}\end{array}$ \\
\hline $\mathrm{q}$ & $1.6 \times 10^{-19} \mathrm{c}$ & $\mathrm{kT} / \mathrm{q}$ & 0.0259 volt \\
\hline
\end{tabular}

S-R-H parameters:

$\tau_{1 \mathrm{t}}=\tau_{2 \mathrm{t}}=\tau_{\mathrm{t}}$ and $\mathrm{n}_{1 \mathrm{t}}=\mathrm{p}_{1 \mathrm{t}}=\mathrm{n}_{\mathrm{i}}$

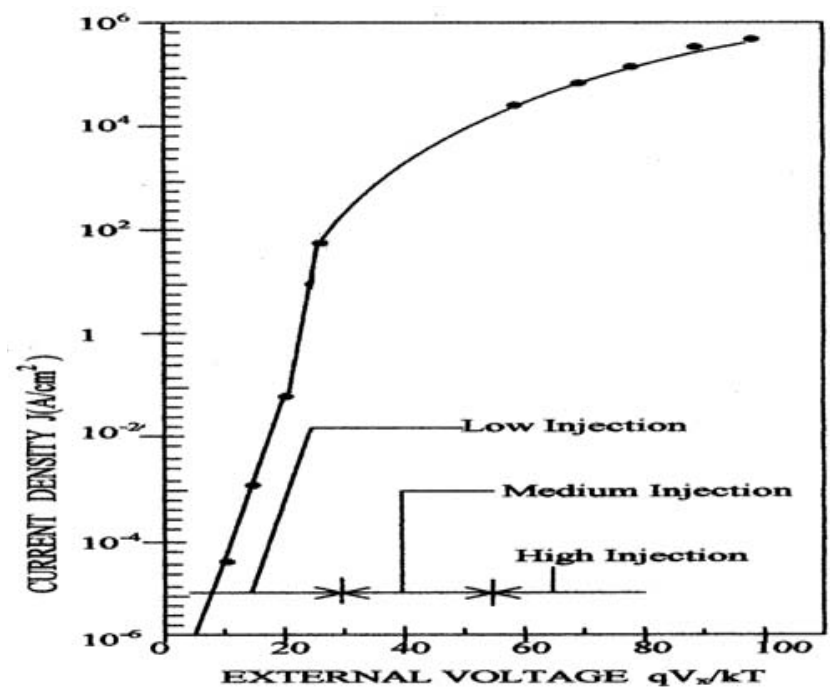

Fig 2: The current as a function of external voltage

Figure 2 indicates the variation of current density as a function of external voltage. It is observed that the current density increases with the increasing value of the external voltage. It is minimum in low injection region and maximum in high injection region. 


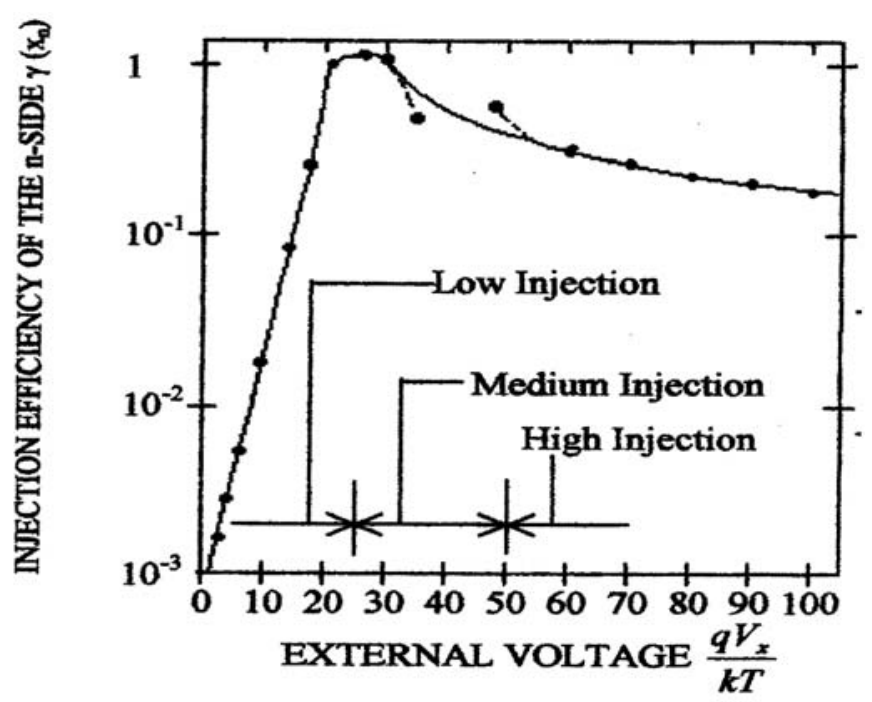

Fig 3: The injection efficiency as a function of external voltage

Figure 3 indicates the variation of injection efficiency with the external voltage. It is observed that the injection efficiency increases with the increasing value of the external voltage. It is chronologically increases with external voltage in low injection region and start to decrease in medium injection region which is maintaining continuity in high injection region.

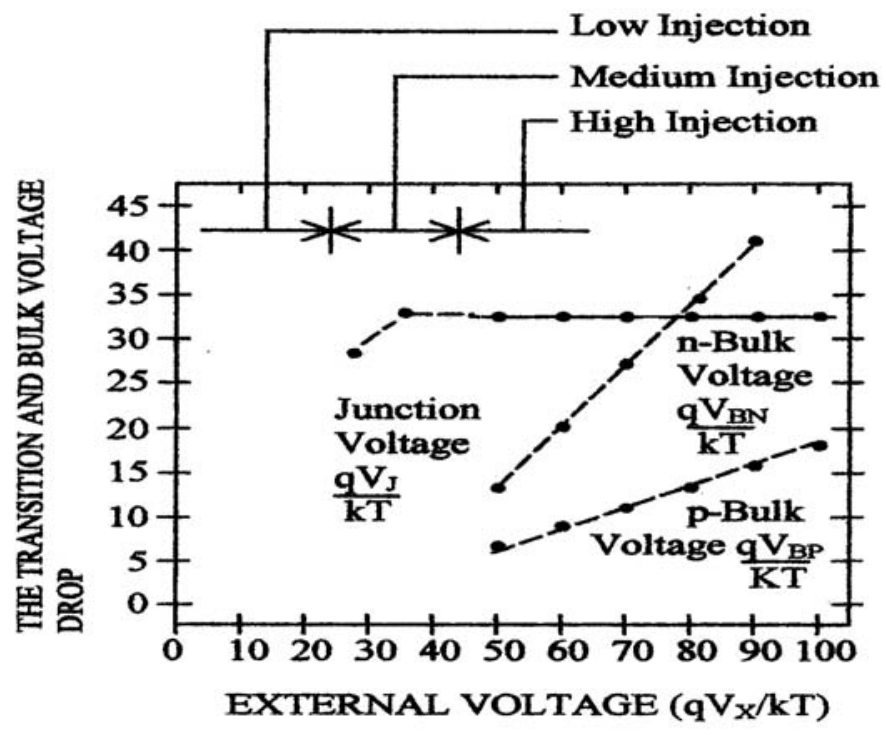

Fig. 4: The transition and bulk voltage drops as a function of external voltage
Figure 4 indicates the variation of transition and bulk voltage drops as a function of external voltage. It is observed that the junction voltage drop, p-bulk voltage and n-bulk voltage drop are not giving response in the low and medium injection region with the increasing value of the external voltage. It also indicates that in high injection region junction voltage drop approximately constant, n-bulk voltage drop rapidly increasing and p-bulk voltage drop chronologically increases with external voltage.

It is interesting to note that the continually decreasing injection efficiency is basically due to the transition region itself. The charge neutrality requirement of the bulk region demands majority carrier increases, which in turn cause a decrease in the injection efficiency. This situation reaches an extreme case in the high injection region and the limiting injection efficiency occurs because the electron and hole mobilities differ.

Finally, it could be conclude that the model can indeed be used as a realistic model for abrupt p-n junctions.

\section{References}

Fletcher N. H. (1957) In high injection conditions, the quasi-Fermi levels separated by an amount larger than the voltage drop $\mathrm{V}_{\mathrm{J}}$ appearing across the junction $J$. Electron. 2: 609-614.

Guckel H., Thomas D. C., Iyengar S. V. and Demirkel A., (1989) The p-n junction transition region Solid-St. Electron 22: 829-834.

Hall R. N. (1952) Physical Review 87: 387-389.

Hazman S. (1967) Solid-St. Electron, l 10: 269-272.

Kasap S. O., (1990-2001) The p-n junction: The Shockley Model, an e-Booklet, by private communication.

Mari De, A. Electron Devices Meeting, (1997) An accurate numerical one-dimensional solution of the P-N junction under arbitrary transient conditions 13: 100-110.

Misawa T. (1966) The Boltzmann factor and the electrochemical potential approach at the injection surface and the pn product of $\mathrm{p}$-n junction must always 
be $\leq n_{i}^{2} e^{\frac{q V}{k T}}$ outside the depletion region Physical Soc. of Japan, 11: 728-732.

Mukul A. (2002) 10 January, Device Physics, by private Communication, 299-239.

Nordman J. E. (1967) High injection of the p-n junction. Solid-St. Electron 10: 263-265.

Nussubaum A. (1978) Boundary conditions for space charge region of a p-n junction Solid-St. Electron 21: 11781182.
Rittner E. S. (1964), Physical review 94: 1161-1163.

Shockley W. and Read W. T. (1952), JR. Physical Review 87: 835-637.

Van Vliet K. M. (1984) High injection theories of p-n junction in the charge neutrality approximation Solid-St. Electron 9: 185-192.

Received : August, 28, 2008;

Accepted : January 28, 2009 